\section{High Resolution 3-D Fluorescence Microscopy: A Comparison of Confocal Laser Scanning Microscopy and a Wide-Field Deconvolution Technique \\ Scanalytics, Inc.*}

\begin{abstract}
Advances in the biological sciences have given rise for the need to visualize microscopic structures of interest. As the requirement to see specific structure and sub-resolution details arise, investigators have turned to fluorescent probes to label and observe these details, which are often undetectable using conventional methods in light microscopy. Fluorescence microscopy offers many advantages for visualizing specific structure and sub-resolution details, which are often undetectable using transmitted light microscopy. Recent advances in fluorescent probe and microscope design, as well as imaging instrumentation designed for fluorescence applications, are now permitting life-science researchers to view details in regions of interest with increasing precision, accuracy, and resolution. Wide field epifluorescence microscopy is presently the easiest and least expensive method for visualizing virtually any fluorescently labeled structure. However, it can be less than optimal for visualizing three-dimensional image volumes, samples heavily labeled with fluorescent dye, or samples that possess fine or weakly labeled detail obscured by interference from signal above and below the image plane of interest. The techniques used to perform high-resolution 3D fluorescence microscopy vary, but emerging as two popular methods are those of confocal laser scanning microscopy and deconvolution of widefield images. Much attention has been given to these approaches over recent years. The goal of this article is to familiarize the reader with both techniques, and allow for an understanding of the application areas in which one may be preferable over the other.
\end{abstract}

\section{SIMPIIFIED OPTICS OF A LSCM}

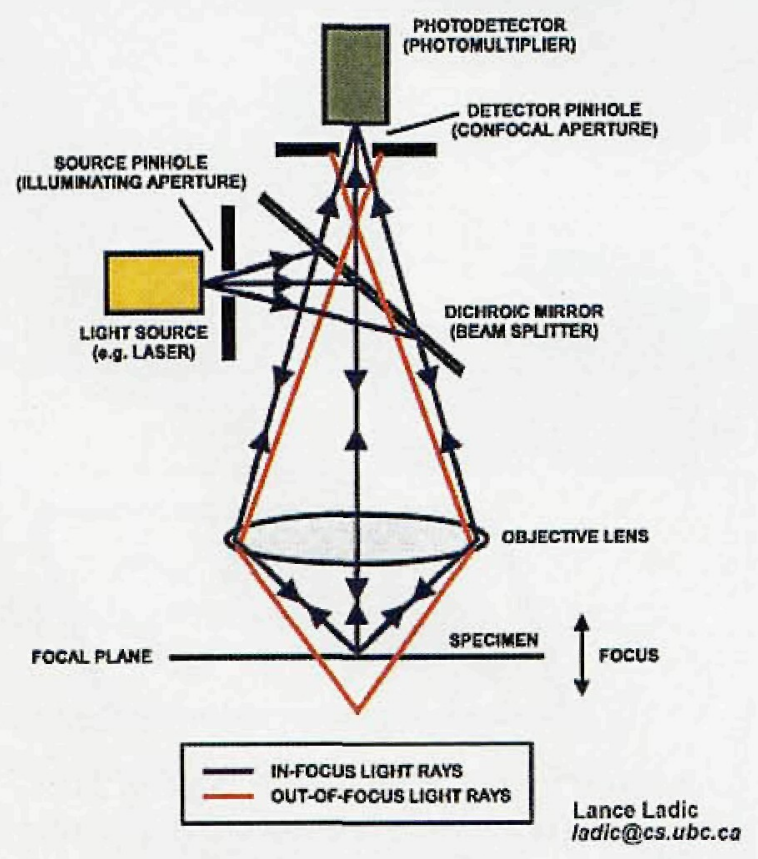

Figure 1: The simplified ray diagram of a confocal laser scanning microscope. in-focus rays (blue) converge from the in-focus image plane and fall on the photomultipler after passing through the pinhole aperture. Out-of-focus rays (red) from focal planes above and below the central plane of focus are blocked from the photomultplier by the mask. (Reproduced with permission from Lance Ladic, University of British Columbia, Vancouver, BC).

\section{Introduction}

Three major difficulties are inherent to fluorescence microscopy. The first involves the very low amount of fluorescent signal available to the image sensor. With even the very best fluorescence microscopes, only a small fraction of the emission wavelength is detectable. Reflection, light scatter and absorption occur at many locations within the optical path of the microscope, decreasing the amount of light available for observation or detection. The second problem concerns out-of-focus haze originating from areas outside the image plane of interest ${ }^{1}$. Signal originating from image planes above and below the plane of focus increase the contribution of light to the specimen image and obscure fine detail that may have been otherwise visible. In addition to these optical concerns, the chemical phenomenon of photobleaching imposes the third difficulty. Photobleaching is the loss of fluorescence signal which results from the chemical degradation of fluorophores. Once exposed to excitation energy, all fluorophors undergo photobleaching. The degree of photobleaching increases as the excitation energy increases ${ }^{2}$. Visually, the effect can be quite dramatic, as structures of interest fade irreversibly - often within a few seconds. Many regions or molecules of interest may be very weakly labeled; a result of low biological expression or physical size hence accommodating only a limited number of fluorescent probes. These particular regions will, as a result, photobleach most rapidly even when illuminated with light levels necessary for detection by an extremely efficient sensing device.

The two predominate methods for addressing many of these problems are confocal laser scanning microscopy (CLSM) and the deconvolution method of Exhaustive Photon Reassignment (EPR). The principles underlying CLSM were first described in 1957, patented in $1961^{\circ}$, and instrumentation has been available commercially since 1985. The deconvolution method of EPR was originally developed in 1979, and received patent protection in 1991. This technique has recently gained importance as biological imaging applications have evolved beyond the capabilities of confocal methods. Scientific-grade cameras and the requisite computer power for the routine use of this technique have also recently become available at a reasonable cost to individual researchers.

\section{METHODS}

\section{Confocal Microscopes}

The confocal microscope addresses the problems of light scatter and out-of-focus haze by placing an adjustable pinhole or slit in the primary image plane of the microscope. The mask allows only light from the focal plane of interest to reach the sensor. Therefore, out of focus light is kept from reaching the detector, increasing both contrast and resolution of image volumes and structures of interest ${ }^{1}$. Illumination of the specimen occurs via a laser ( $\mathrm{HeNe}, \mathrm{KrAr}$ ) and a photomultiplier tube is most typically used as a detector. The laser point is scanned over the specimen using a series of galvonometrically-controlled mirrors and photons are collected by the photomultiplier tube at each scan-point. Image volumes may be created by acquiring image planes at several optical depths through the use of a focusing device coupled directly to the fine focus knob. The microscope from which the image is collected is normally equipped with high magnification, high numerical aperture, long working distance objective lens. Standard computers and commercial software packages permit the display and analysis of resulting images.

This method has several important advantages over other methods of high resolution fluorescence microscopy. Laser light is much more powerful than arc lamp illumination, so imaging can be performed deep into tissue sections. Since out of focus signal is rejected by the pinhole, samples that are heavily labeled appear clear in the confocal microscope. It is also one of the fastest ways to get a single, haze-free image plane of a given specimen volume. Since its commercial introduction in the mid 1980's, the confocal microscope has become a key instrument in many core facilities, and hundreds of papers have been published using this technique.

Several problems are associated with pinhole confocal microscopes. Lasers have traditionally been an inflexible light source, featuring only a few wavelengths that can be used for exciting fluorophores. Since a pinhole has been placed in the light path, a laser is the only method to generate enough emission signal to reach the detector. FURA-2 and standard nuclear counterstains such as DAPI and Hoechst have been problematic dyes to image without an expensive UV laser. In recent years, tunable lasers which feature UV capabilities are 
coming down in price, but are still prohibitively expensive in many cases. Lasers have other drawbacks, such as causing severe photodamage and decreasing the time it takes for a specimen to photobleach.

Photon efficiency for CLSM system has been reported to be less than $1 \%{ }^{3}$. Pinhole confocal microscopes, therefore, usually have scan times of $2-40$ seconds, depending on the desired quality of final image. Short scan times often result in images that are poorly resolved, while movement of the specimen during a longer scan time poses problems for imaging dynamic events over time. In addition, living specimens often react poorly to continued illumination with laser light.

Quantitation of intensity values presents many challenges for confocal microscopy, but experimental methods have been devised to permit their use in some quantitative studies ${ }^{4}$. The photomultiplier tube is non-linear in its detection of photons. This situation is exacerbated by the photobleaching caused by the laser.

Confocal microscopes typically require management by a sophisticated knowledgeable user. Many confocal manufacturers have addressed these concerns in recent years, and they have automated many of the settings. While this increases the ease of use for most researchers, these instruments still require a great deal of maintenance, and a dedicated professional is usually charged with day-to-day operation of the instrument.

One method of increasing the amount of signal detected is to decrease the amount of signal rejected. The use of a slit in the primary image plane, rather than a pinhole allows a swath of light to be scanned over the specimen, and hence a greater volume of light is allowed to reach the detector. This has several advantages over a pinhole confocal microscope. First, it increases the detection of faint or rapidly photobleaching signals, since more light is allowed to pass to the detector. Second, scan times are greatly reduced, so living specimens are more easily imaged with this type of confocal. Slit confocal microscopes also require lasers, so they continue to have problems with photobleaching and photodamage. Finally, since a slit confocal microscope allows more light to pass to the specimen and to the detector, resolution is sacrificed due to an increase in out-of-focus light remaining in the images

\section{Deconvolution}

Deconvolution describes many image and signal processing algorithms with applications in fields as diverse as astronomy, seismology and life science imaging. Discussed here is a rigorous and patented method of deconvolution known as Exhaustive Photon Reassignment (or EPR) developed and optimized to produce quantitatively correct, high contrast, high resolution images from volumes acquired through wide-field fluorescence microscopes". This algorithm characterizes the optical properties of fluorescence microscopes, then applies

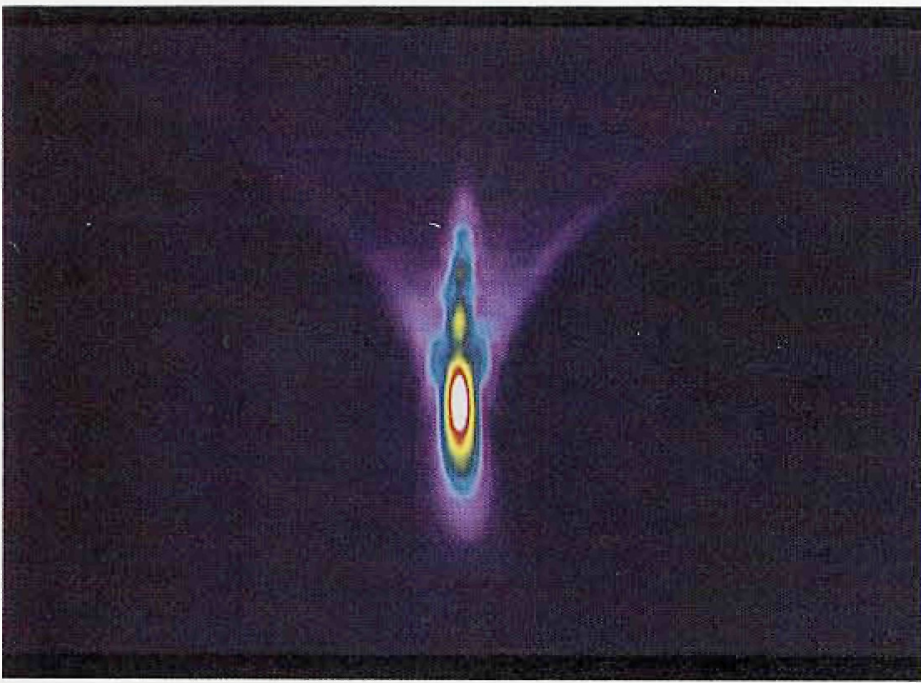

Figure 2. Point-spread flinction (PSF) viewed as an $x / z$ orthogonal projection and pseudocolored to show relative intensity levels. The white central portion of the PSF represents a sub-resolution microbead (100 nm dia.) labeled with Nile Red (Molecular Probes, Eugene, OR). Signal measured above and below the central planes represents the optical characteristics of an Olympus 60X Plan Apochromat 1.4 NA objective lens this characterization to reverse the optical properties. The effect of the process is to reassign out-of-focus light back to its original locations within (or outside of) the image volume and to reverse many of the aberrations introduced by the optics 5 .

The EPR algorithm makes use of the assumption that the microscope is a linear system: an image is formed from the sum of it's parts, and light passing through the elements of the microscope is distorted equally, regardless of its position within the field of view. A characterization of this phenomenon may be created by examining a point source, typically a fluorescently-labeled microbead, at varying optical depths. Acquiring image planes at sequential optical depths above and below the microbead's central plane of focus yields a distinctive hourglass shape describing the performance of the optical path. This blurring function, commonly known as the 'pointspread function' (PSF) is then used by the algorithm as a 3-D vector to reassign out-of-focus signal to its correct locations within the image volume.

EPR deconvolution does have several disadvantages. First; the image after processing is only as accurate as the point-spread function that was applied to the image set. As with other scientific measurement tools, if the calibration standard is not acquired under representative conditions, the end result is not reliable. Second, although the efficiency of the technique permits image volumes to be acquired extremely rapidly, the deconvolved results often take several minutes to process. High-quality unprocessed images are available real-time during acquisition, but EPR processing is generally performed off-line. Third, the technique of EPR deconvolution cannot be performed on specimens which have been labeled to the point where the instrument is saturated with fluorescence signal. This situation occurs when extremely thick, dense specimens have been heavily labeled with a nonspecific fluorescent probe. Specimens of this type, however, are often not intended for especially high-resolution applications, are almost always fixed (not living), and are therefore ideal for confocal imaging. Interestingly, additional resolution can almost always be gained from confocal images, by applying EPR to confocally-derived images.

The key benefit to the use of this method is that image volumes are acquired through conventional fluorescence microscopes. As there are no apertures introduced to exclude signal from detection, the total system is much more efficient and sensitive. This efficient collection and use of signal information leads to the achievement of spatial resolution exceeding the classical Rayleigh definition by as much as four times ${ }^{6,7}$. Illumination of the specimen occurs through standard Mercury or Xenon arc-lamps using filter sets optimized for the probes being used. As shown in Figure 2, the use of

\section{Continued on Page 12}

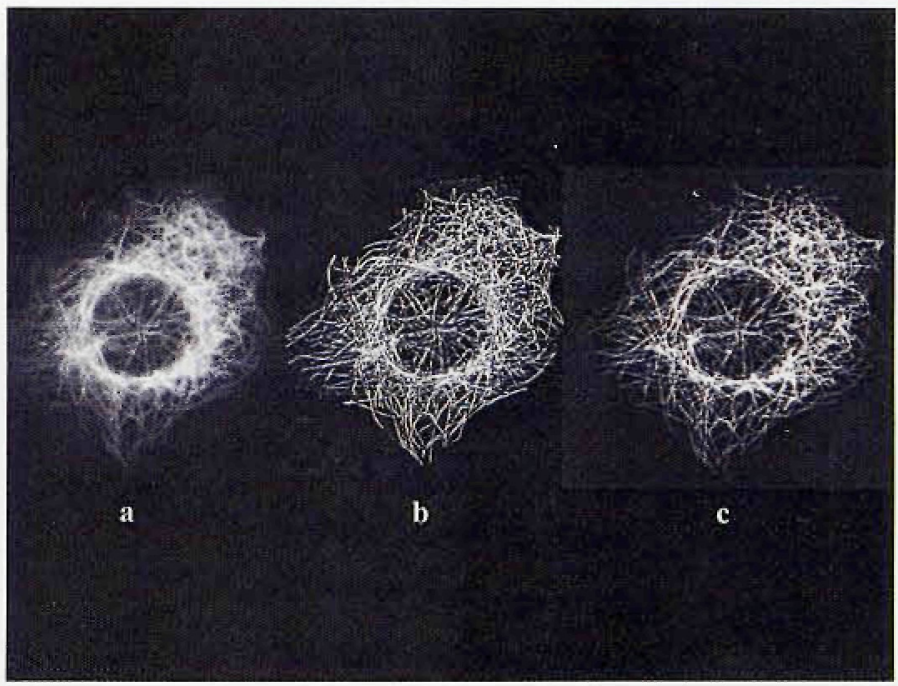

Figure 3. Normal Rat Kidney (NRK) epithelial cell with rhodamine-labeled tubulin. Images represent volumes acquired with (a) standard wide-field epifluorescence microscope, (b) standard wide-field epifluorescence microscope and restored using the Exhaustive Photon Reassignment (EPRTM) alogorithin developed and patented by the Biomedical Imaging Group of the University of Massachusetts Medical School (available commercially by Scanalytics (Billerica, MA)), and (c) BioRad MRC 600 confocal microscope. 
High Resolution 3-D Fluorescence Microscopy. . . Continued from Page 11

such broad spectrum illumination allows researchers to excite virtually any fluorophore. High magnification, high numerical aperture objective lenses are used to acquire image volumes. Fluorescent signal is detected with commercially available charge-coupled device (CCD) cameras, selected for their linearity, dynamic range, high quantum efficiency, and perfect geometric accuracy ${ }^{11}$. Image volumes may be created by acquiring image planes at varying optical depths. Images are typically acquired, displayed, and analyzed on a variety of standard computer platforms. Processing of images using the EPR algorithm requires the use of a high-end computer (e.g., PentiumPro@) or optimized mathematical accelerator for high throughput of very large image sets.

\section{Discussion}

A great deal of discussion has come about in recent times concerning the absolute advantage of one technique over the other. While each technique attempts to address the problems inherent to performing highresolution fluorescence microscopy, they do so by very different methods. Each method derives from a distinct set of research needs and therefore emphasizes a different paradigm of the problem. The chief source of confusion between the two techniques is that both methods result in thin optical sections of fluorescently-labeled microscopic specimens. The similarity, however, ends there. Confocal microscopy is built on the premise that out-of-focus signal reduces spatial resolution and therefore must be physically rejected from the optical system. Confocal systems, therefore, are designed to occlude signal from all but a thin focal plane. Imaging of dim and bleaching fluorescent specimens, therefore, can be performed only slowly and briefly. EPR deconvolution is built on the premise that out-of-focus signal contains valuable information. This information can be, with the assistance of a powerful computer, sorted out and placed back to where it originated. EPR-based systems, therefore, are designed to optimize the collection of signal from the specimen volume, resulting in a method which is extremely sensitive, gentle, and fast. Depending on one's specific imaging challenges, one method may be preferred over the other as shown in the table below.

\section{Imaging Requirement}

Extremely Thick/Dense Specimens

Real-Time Viewing of Thin Optical Sections

Fixed (Dead) Specimens

UV-Excited Fluorophores

Living/Delicate Specimens

Quantitation of Instensity Values (e.g. Ratiometry)

Viewing in 3D Over Time (one or more wavelengths

Fast Imaging of $3 \mathrm{D}$ Voumes

Extremely High Resolution in $X Y$ and $Z$

\section{Convenience}

Probably the most important, non-technical, issue concerning the two methods is that of convenience. User interaction and sophistication with each method will have a major impact on how reliable and reproducible the resulting images will be.

To acquire accurate images through the CLSM, the laser must be both accurately aligned and emit appropriate wavelengths to stimulate fluorescence. Very high quantum efficiency may be obtained when a laser is properly matched to a fluorescent probe, but will decrease dramatically if the laser and probe possess different excitation spectra characteristics. Once the laser has been selected and aligned, scanning of the sample can occur and with the user-friendly software that is commercially available, is a straightforward process. In the past, aligning the laser to properly illuminate specimens had proven difficult, but advances in technology have improved these methods, reducing the need for a highly trained, sophisticated user to attain accurate and reproducible images. Selection of the laser to stimulate fluorescence is also critical.

Acquiring accurate images for Exhaustive Photon Reassignment processing can be more complicated. The principal concern to users of EPR is obtaining an accurate point-spread function (PSF). The PSF must take into account, and mirror exactly, the optical properties of the microscope being used. Once accurate PSFs have been obtained that reflect various objectives, immersion and mounting media, and fluorochromes, they may be stored as image files in a library on a computer disk. As with CLSM software, user-friendly software is available for users to perform deconvolution routines and subsequent image analysis.

\section{Summary}

The two most prevalent techniques available for performing high-resolution 2-D and 3-D fluorescence microscopy-confocal laser scanning, and deconvolution of wide-field images - were developed to solve the problems inherent to conventional fluorescence microscopy. Each method presents a suite of strengths and weaknesses, with their overlap being the cause of much discussion. Life science researchers using fluorescence microscopy who desire a better understanding of cellular structure and function will be best served by understanding and evaluating the two methods prior to making a purchase.

1) Inoue, S., 1995. Confocal Scanned Images and Light Microscopy. In: Handbook of Biological Confocal Microscopy, (J. Pawley, ed.), Plenum Press, pp. $1-17$.

Preferable Method Confocal EPR Deconvolution $\checkmark$ $\checkmark$

2) Tsien, R.Y. and A.S. Waggoner, 1990. Fluorophores for confocal microscopy Photophysics and Photochemistry. In: Handbook of Biological Confocal Microscopy. (J. Pawley, ed.), Plenum Press, pp.267-274

3) Wells, K. Sam, David R. Sandison, James Strickler, Watt W. Webb, 1990, Quantita tive Fluorescence Imaging with Laser Scanning Confocal Microscopy. In Confocal Microscopy Handbook; (J. Pawley, ed.), IMR Press, pp. 27-39

4). Art, Jonathan J. and Miriam B. Goodman. Rapid Scanning Confocal Microscopy. In: Methods in Cell Biology, Vol.38. (Brian Matsumato, ed.). 1993, Academic Press, pp.47-77.

5). Carrington, W.A., Image Restoration in 3D Microscopy with Limited Data. SPIE Proceedings, 1990, 1205: pp.72-83

6). Carrington, Walter A., Ronald M. Lynch, Edwin D.W. Moore, Gerrit Isenberg, Kevin E. Fogarty, Frederic S. Fay. 1995. Superresolutionthree-dimensional images of fluorescence in cells with minimum light exposure. Science, 268: 1483-1487.

7). Carter, Kenneth $C$., Douglas Bowman, Walter Carrington, Kevin Foga rty, John A. McNeil, Fredeic S. Fay, Jeanne Bentley Lawrence. 1993. A three dimensional view of precursor messenger RNA metabolism within the mammalian nucleus. Science. 259: 1330-1335.

8). Minsky, M. (1961). U.S. Patent 3,013,467.

9). Carrington, W.A., Kevin E. Fogarty, Larry Lifschitz, Frederic S. Fay. 1990. 3D Imaging on Confocal and Wide Field Microscopes. In: Confocal Microscopy Handbook, (J. Pawley, ed.), IMR Press, pp.151-161.

10). Lansing Taylor, D. and E.D. Salmon. 1989. Basic Fluorescence Microscopy. In: Fluorescence Microscopy in Living Cells in Culture - Part A. (Y.L. Wang and D. Lansing Taylor, eds.), Academic Press, pp.223.

11). Spring. K., 'Detectors for Fluorescence Microscopy'. Course Notes from Optical Microscopy and Imaging in the Biomedical Sciences'. Marine Biological Laborato ries, Woods Hole, MA (1994).

* Readers wishing more information on this subject may contact at Scanalytics: Jennifer Robinson at (508)663-8161 (scan_info@cspi.com) or Paul Krumpe at (703)281-3277 (info@iplab.com) 


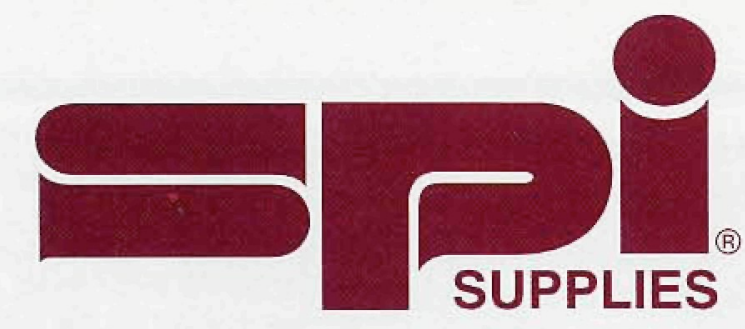

है File Edit Uiew Go Bookmarks 0ptions Directory Help

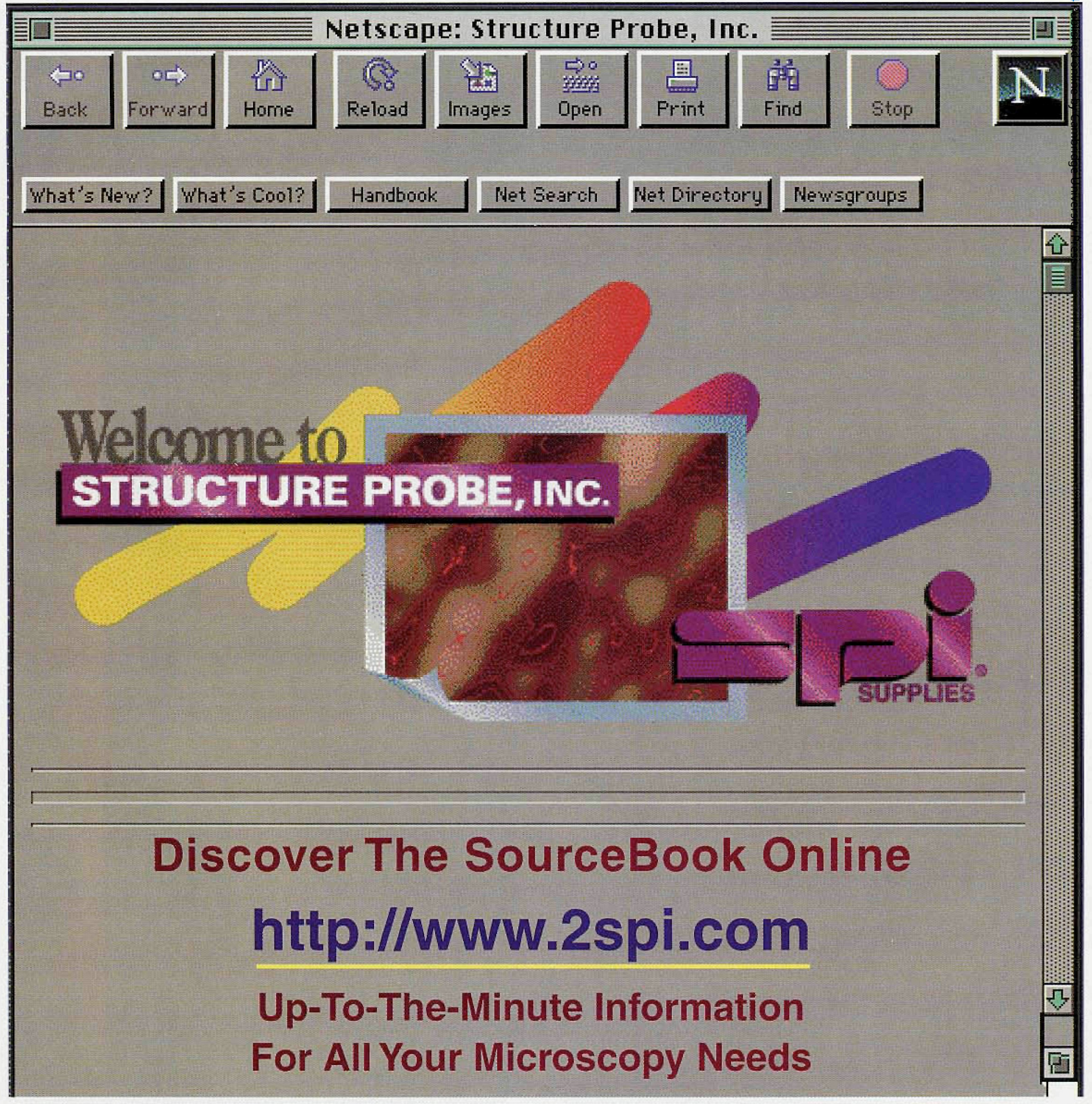

SPI Supplies Division of STRUCTURE PROBE, Inc.

P.O. Box 656 - West Chester, PA 19381-0656 USA 